\title{
A COUNTEREXAMPLE TO A RESULT ON LOTKA-VOLTERRA SYSTEMS
}

\author{
JAUME LLIBRE
}

\begin{abstract}
In the article of "Acta Applicandae Mathematicae $\mathbf{2 3}$ (1991), 103-127" the authors claim the existence of a Hopf bifurcation which in general does not exist.
\end{abstract}

\section{InTRODUCTION AND THE COUNTEREXAMPLE}

In [1] the generalized Lotka-Volterra model of the form

$$
\begin{aligned}
& \dot{x}=x^{\hat{p}(\mu)}-x^{p(\mu)} y^{q(\mu)}, \\
& \dot{y}=C(\mu)\left(x^{p(\mu)} y^{q(\mu)}-y^{\hat{q}(\mu)}\right),
\end{aligned}
$$

with $C(\mu)>0$ is analyzed. The authors claim to have the following result.

Theorem 1. Assume that the functions $\hat{p}(\mu), p(\mu), \hat{q}(\mu), q(\mu)$ and $C(\mu)$ are continuously differentiable and for admissible values of $\mu$ these functions are positive and satisfy

$$
\hat{p} q+p \hat{q}-\hat{p} \hat{q}>0 .
$$

If, for some $\mu_{0}$,

$$
\hat{p}\left(\mu_{0}\right)-p\left(\mu_{0}\right)=C\left(\mu_{0}\right)\left(\hat{q}\left(\mu_{0}\right)-q\left(\mu_{0}\right)\right)
$$

and

$$
\left.\frac{d}{d \mu}(\hat{p}-p-C(\hat{q}-q))\right|_{\mu=\mu_{0}} \neq 0,
$$

then the system undergoes an Andronov-Hopf bifurcation at $\mu_{0}$. Moreover, the bifurcation is supercritical, resp. subcritical according to

$$
p(\hat{p}-p)(\hat{q}-1)(\hat{q}+q)-\left.q(\hat{q}-q)(\hat{p}-1)(\hat{p}+p)\right|_{\mu=\mu_{0}}<0,
$$

resp. $>0$.

Unfortunately there is a problem in the proof of Theorem 1 due to the following counterexample.

2010 Mathematics Subject Classification. 37K10, 37C27, 37K05.

Key words and phrases. Lotka-Volterra system, Hopf bifurcation. 
Theorem 2. Consider the polynomial differential system

$$
\begin{aligned}
& \dot{x}=x-x^{2} y^{2}=P(x, y), \\
& \dot{y}=(1+\mu)\left(x^{2} y^{2}-y\right)=Q(x, y),
\end{aligned}
$$

with $1+\mu>0$. System (5) satisfies all the assumptions of Theorem 1 with $\mu=\mu_{0}=0$ but it does not exhibit an Andronov-Hopf bifurcation.

Proof. Comparing system (1) with system (5) we have

$$
\hat{p}(\mu)=\hat{q}(\mu)=1, \quad p(\mu)=q(\mu)=2, \quad C(\mu)=1+\mu .
$$

Then we have

$$
\hat{p} q+p \hat{q}-\hat{p} \hat{q}=3>0 .
$$

So, condition (2) holds.

Take $\mu_{0}=0$. Then, condition (3) is immediately satisfied, and for condition (4) we obtain

$$
\left.\frac{d}{d \mu}(\hat{p}-p-C(\hat{q}-q))\right|_{\mu=0}=1 \neq 0 .
$$

In short, all the conditions of Theorem 1 are satisfied by system (5).

The unique equilibria of system $(5)$ are the $(0,0)$ and $(1,1)$. Around the equilibrium point $(0,0)$ cannot exist periodic orbits because the straight lines $x=0$ and $y=0$ are invariant by the flow of system (5), i.e. they are formed by orbits of system (5). Therefore, if there are periodic orbits these must surround the equilibrium point $(1,1)$. We recall that in the bounded region limited by a periodic orbit of a differential system in the plane it must be an equilibrium point, see for instance Theorem 1.31 of [2].

We claim that the unique periodic orbits of systems (5) for all $\mu$ exist when $\mu=0$, and they are the periodic orbits surrounding the center $(1,1)$ of system $(5)$ with $\mu=0$. Now we shall prove the claim. Clearly once the claim is proved it follows that system (5) cannot exhibit an Andronov-Hopf bifurcation.

System (5) with $\mu=0$ has the first integral $H=x+y+1 /(x y)$, because the derivative of $H$ on the orbits of system (5) with $\mu=0$ satisfies that

$$
\frac{d H}{d t}=\frac{\partial H}{\partial x}\left(x-x^{2} y^{2}\right)+\frac{\partial H}{\partial y}\left(x^{2} y^{2}-y\right)=0 .
$$

Since the eigenvalues of the linear differential system (5) with $\mu=0$ at the equilibrium $(1,1)$ are $\pm \sqrt{3} i$, this equilibrium either is a focus or a center, but it cannot be a focus because the first integral $H$ is defined at $(1,1)$. Hence, we have proved that the equilibrium $(1,1)$ for system 


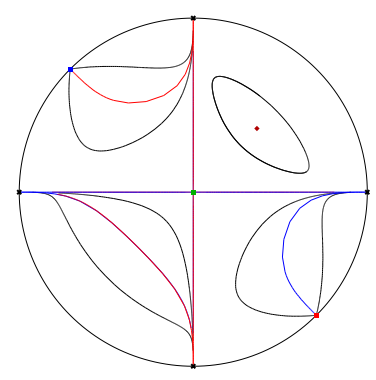

FiguRe 1. Phase portrait of system (5) with $\mu=0$.

(5) with $\mu=0$ is a center. Now we shall see that the periodic orbits of this center filled all the positive quadrant $Q=\{(x, y): x>0$ and $y>$ $0\}$. Assume that they do not filled all that quadrant. Since in that quadrant the unique equilibrium is the $(1,1)$, the external boundary of the continuum set of periodic orbit surrounding the center $(1,1)$ must be a periodic orbit $\gamma$, and after that orbit the nearby orbits must spiral. Consider the Poincaré map defined in an analytic transversal arc $\Sigma$ to this periodic orbit $\gamma$. Since the flow of the polynomial differential system (5) with $\mu=0$ is analytic, such a Poincaré map is analytic, but it is not possible that an analytic map of one variable be the identity on the piece of the arc $\Sigma$ contained in the bounded region limited by $\gamma$, and different to the identity on the piece of the arc $\Sigma$ outside the bounded region limited by $\gamma$. So such a last periodic orbit $\gamma$ does not exist and the periodic orbits surrounding the center $(1,1)$ filled all the positive quadrant $Q$. See a picture of the phase portrait of system (5) with $\mu=0$ on the Poincaré disc in Figure 1, for more details on the Poincaré disc see Chapter 5 of [2].

For completing the proof of the claim we must show that system (5) with $\mu \neq 0$ has no periodic solutions surrounding the equilibrium $(1,1)$. We shall use the Dulac criterium: Let $P$ and $Q$ be the polynomials defined in (5). If there exists a $C^{1}$ function $B(x, y)$ in a simply connected region $R$ such that $\partial(B P) / \partial x+\partial(B Q) / \partial y$ has constant sign and is not identically zero in any subregion of $R$, then system (5) does not have a periodic orbit lying entirely in $R$. For a proof of this criterium see for instance Theorem 7.12 of [2].

Consider the function $B=1 /\left(x^{2} y^{2}\right)$ defined in the positive quadrant $Q$. Then

$$
\frac{\partial(B P)}{\partial x}+\frac{\partial(B Q)}{\partial x}=\frac{\mu}{x^{2} y^{2}} \neq 0 \text { in } Q \text { if and only if } \mu \neq 0 .
$$


Therefore, by the Dulac criterium, system (5) with $\mu \neq 0$ has no periodic solutions surrounding the equilibrium $(1,1)$, and this prevents the existence of a Hopf bifurcation. The proof of the claim and of Theorem 2 is completed.

\section{ACKNOWLEDGEMENTS}

The first author is partially supported by a MINECO/FEDER grant MTM2008-03437, a CIRIT grant number 2009SGR-410, an ICREA Academia, two grants FP7-PEOPLE-2012-IRSES 316338 and 318999, and UNAB13-4E-1604.

\section{REFERENCES}

[1] A. Dancsó, H. FArkas, M. FArkas and G. Szabó, Investigations into a class of generalized two-dimensional Lotka-Volterra schemes, Acta Appl. Math. 23 (1991), 103-127.

[2] F. Dumortier, J. Llibre And J.C. Artés, Qualitative theory of planar differential systems, Springer-Verlag, New York, 2006.

Departament de Matemátiques, Universitat Autónoma de Barcelona, 08193 Bellaterra, Barcelona, Catalonia, Spain

E-mail address: jllibre@mat.uab.cat 